\title{
BIBLIOGRAPHY OF SURFACES AND TWISTED CURVES.
}

BY DR. J. E. HILL.

The following article gives a brief sketch of a bibliography of surfaces and twisted curves, prepared by the writer, and contains some miscellaneous notes pertaining to the general subject. It comprises for the most part extracts from a paper read before the American Mathematical SocIETY at its last May meeting.

The bibliography is intended to represent a compilation and classification, as complete as possible (with certain exceptions), of all articles upon surfaces and twisted curves published during the present century. The exceptions noted above are the general discussion of quadric surfaces and sphero-conics and the complete consideration of line geometry. References to papers upon the curvature, lines of curvature, geodesios, umbilics, and general surface curves of quadric surfaces have been included and classified ; but, otherwise, articles upon the subjects whose exclusion has been mentioned are listed only incidentally.

The bibliography is indexed, subject to revision, as follows: 1. Surfaces in general. 2. Curvature of surfaces; (a) of quadrics, (b) in general. 3. Lines of curvature; (a) upon quadrics, (b) in general. 4. Geodesics; (a) upon quadrics, (b) in general. 5. Surfaces curves; (a) upon quadrics, (b) in general. 6. Umbilics. 7. Contact of surfaces. 8. Curvilinear coördinates. 9. Algebraic surfaces. 10. Cubic surfaces. 11. Quartic surfaces; (a) in general, (b) the wave surface, (c) the torus, (d) the cyclide, (e) Kummer's surface, (f) Steiner's surface, (g) the surface of elasticity. 12. Surfaces of the fifth and higher orders; (a) quintic surfaces, (b) sextic surfaces, (c) septic surfaces, (d) octic surfaces, (e) 9-thic surfaces, (f) 10-thic surfaces, (g) 12-thic surfaces. 13. Surfaces of revolution. 14. Orthogonal surfaces. 15. Envelopes. 16. Reciprocal surfaces. 17. Minimal surfaces. 18. Ruled surfaces; (a) in general, (b) scrolls, (c) torses. 19. Surfaces defined by curvature; (a) surfaces of constant curvature, (b) surfaces with plane lines of curvature, (c) surfaces with spherical lines of curvature, (d) other special forms. 20. Special forms of surfaces; (a) applicable, (b) helicoidal, (c) isothermal, (d) parallel, (e) normal, (f) polar, (g) pseudo, (h) pedal, (j) cyclic, (k) flexible and inextensible, (1) derived, (m) focal, 
(n) anallagmatic, (o) apsidal, (p) inverse, (q) other unclassified forms. 21. 'Twisted curves in general. 22. Curvature of twisted curves. 23. Algebraic twisted curves. 24. Twisted cubics. 25. Twisted quartics; (a) in general, (b) of the first kind, (c) of the second kind. 26. 'Twisted curves of the fifth and higher orders; (a) twisted quintics, (b) twisted sextics, (c) twisted septics, (d) twisted octics, (e) twisted 10-thics. 27. Other special, unclassified forms of twisted curves.

The bibliography, in its present form, consists of 3,715 different references. Each reference gives the complete title of the paper listed, the name of the author, the year and the journal of publication, the number of the journal and the number of the pages occupied by the article. At the end of each section, cross-references to articles of other sections are given.

Section 1, devoted to surfaces in general, contains 489 references, besides cross-references to some sixty other articles; the dates of publication of the papers referred to extend from 1784 to 1896 . In order that this section, containing such a large number of titles, should be readily accessible to reference, an index has been appended to the section, enumerating the following divisions: Generation, Intersection, Deformation, Quadrature, Singularities and Transformation (including Representation, Projection and Correspondence). All articles of this section not included in these divisions group themselves under the very convenient head of General Theory.

It is interesting to note the names of the mathematicians who, during the first half of this century, concerned themselves with the subjects comprised in this section. Chief among such may be mentioned Monge, Lamé, Crelle, Gauss, Binet, Specht, Olivier, Gudermann, Plücker, Möbius, Tortalini, Löwestern, Gregory, Cauchy, Steiner, Bedetti, Bertrand, Bonnet, Umpfenbach, Ellis, Amiot, Schläfli, Bouquet, Salmon, Bravais, Joachimsthal, Lebesgue, Hachette, Gergonne, Fregier, Bobillier, Hamilton, Conti, Dirksen, Brunn, Boole, and Chelini; some of these are the names of men already forgotten. The first reference in this section is that to a fourteen page article, by Monge, "Sur l'expression analytique de la génération des surfaces courbes," published in the first volume of the Mémoires de l'Académie des Sciences de Turin, 1784.

The first article, listed in section 2, is a six-page paper, by Hachette, published in 1804 in the Correspondance sur l'École Polytechnique and entitled "De quelques pro- 
priétés des rayons de courbure d'une surface." 'This section contains 193 references and is introduced by references to articles by Hachette, Binet, Gergonne, Rodrigues, Sturm, Schubert, Plücker, Sophie Germaine, Poisson, Minding, Transon, Fink, Chelini, Paignon, Thomson, Joachimsthal, Babinet, Liouville, Bonnet, Breton, Dienger and Lebesque. The majority of these names, it will be noted, are French; in fact, in this section, as well as in section 1, nearly every article referred to, appearing during the first quarter of this century, was written by some French mathematician. As a matter of fact, Meusnier read, his celebrated "Mémoire sur la courbure des surfaces" before the French Academy of Sciences in February, 1776, and for the next fifty years this subject was an enthusiastic one for the French school. The first reference to the curvature of quadrics, specifically treated, is that to a paper by Dupin, published in 1804 in the last-mentioned journal ; it is entitled "Détermination des rayons de courbure des surfaces du second degré."

In referring to the lines of curvature of surfaces, the first article noted is the 20-page paper of Monge in the Journal de l'École Polytechnique, 1795, entitled "Sur les lignes de courbure de la surface de l'ellipsoide." The first English article listed is that of Lardner, in the Transactions of the Irish Academy, 1824, volume 14, being "An investigation of the lines of curvature of ellipsoids, hyperboloids and paraboloids." The English mathematicians early devoted themselves to the study of this branch of the theory of surfaces, the names of Ellis, Cayley, and Thomson frequently appearing, while the name of Michael Roberts is one inseparably connected with the history and study of the lines of curvature of surfaces. There are 78 references in this section of the bibliography.

The study of geodesics is, of course, most closely connected with that of the general line of curvature and most articles upon the one deal with the other. The first article, however, that I note as treating specifically of geodesics is Euler's "Accuratior evolutio problematis de linea brevissima in superficie quacunque ducenda," first published in the Nova Acta Academice Scientiarum Petropolitance, 1806, volume 15. Of the English mathematicians, James Ivory appears to have been the first actually to contribute to the theory of geodesics, and his article in the 67th volume of the Philosophical Magazine, "On the properties of the lines of shortest distance traced on the surface of an oblate spheroid," is the first article of the century expressedly investigating this subject from the standpoint of the quadric sur- 
face. The papers of Jacobi, Joachimsthal, Liouville, Chasles, Gauss and Michael Roberts are to be especially mentioned. What was written of this last distinguished mathematician in the last paragraph, holds rigorously true under this. The bibliography contains 135 direct references to articles upon geodesics.

The general surface curve is listed independent of any relation it may bear to the curvature of the surface and has, on this account, an inseparable relation to the intersection of surfaces and the general twisted curve; I have placed it in a section by itself, however, because I deemed that the number of cases in which it has been treated, in ipso, demanded that I should do so. There are 104 references to this section and the first reference is to a paper by Legendre, "Analyse des triangles tracés sur la surface d'un spheroid," published in the sixth volume of the Mémoires de la classe des sciences mathématiques et physiques de l'Institut, 1806.

I am able to find but twenty papers treating specifically of umbilics, the first reference being to the paper of Monge and Hachette, "Application d'algebra à la géomètrie," in the fourth volume of the Journal de l'École Polytechnique, 1802. While this paper does not actually treat of umbilics, it deserves its place at the head of the list of papers upon this subject in that it is the first paper in which the discussion of the conditions for the circular sections of quadrics is undertaken and the idea of umbilics broached. Although there are but twenty references, some of the papers referred to are worthy of much consideration, e. g., those by Verdam, 1852 in the fifth volume of the Tijdschrift voor de Wis- en Natuurkundige Wettenschappen; by Voss, 1875, in the ninth volume of the Mathematische Annalen; and by Servais, 1885 , in the eighteenth volume of the third series of the Bulletin de l'Académie des sciences, des lettres et des beaux-arts de Belgique.

The first paper treating manifestedly of the contact of surfaces is Hachette's "De la courbe de contact d' une surface conique avec une surface dont l'equation est du degré $m$," in the first volume of the Correspondance sur l'Ecole Polytechnique, 1804. Of the twenty-six references listed in this section, those to the papers of Spottiswoode, extending through the '70's, are especially noticeable.

Gauss first introduced the general theory of curvilinear coördinates in his celebrated memoir, "Disquisitiones circa superficies curvas," published in 1823 and translated into French in the ninth volume of the first series of the Nouvelles Annales de Mathématiques, 1852. The first English 
paper that I find is Gregory's "On the method of spherical coördinates," published in the first and second volumes of the Cambridge Mathematical Journal, 1839. This section contains thirty-nine references; and the well-known paper of Lamé, "Léçons sur les coördonnées curvilignes," Paris, 1859, and the lengthy memoir of de Salvert in the $A n$ nales de la Société scientifique de Bruxelles, 1884, are particularly noticeable.

Section 9, upon algebraic surfaces, which is introduced to abbreviate section 1 and fittingly to prepare the way to references to articles upon surfaces of determinate orders, is headed by Lardner's, "On the general properties of algebraic surfaces," published in the 14th volume of the Transactions of the Irish Academy, followed by Plücker's historic paper, "Récherches sur les surfaces algébriques des tous les degrés," appearing in the 19th volume of the Annales de Mathématiques pures et appliquées (Gergonne). There are 271 references in this section.

It is remarkable that the first paper that $I$ can find that deals specifically with the cubic surface is one by L. Mossbrugger, "Untersuchungen über die geometrische Bedeutung der constanten Coefficienten in den allgemeinen Gleichungen der Flächen des zweiten und dritten Grades." This paper appeared in the first volume of the Archiv der Mathematik und Physik, 1841. Salmon's and Cayley's papers upon the triple tangent planes of surfaces of the third order, 1849, published in the fourth volume of the Cambridge and Dublin Mathematical Journal, are the first English papers listed in this section. Although one may say that the classification of cubic surfaces is practically complete, the study of these surfaces appears, still to-day, to have the same fascination as was exhibited when the discovery of the existence and the relations of the 27 lines of the general cubic surface was first announced, no less than a round dozen of worthy articles upon cubic surfaces appearing last year. Of the 205 articles referred to in this section, nearly all are worthy of close perusal.

Although the first reference to the quartic surface, treated generally dates no farther back than 1848, being Poncelet's article in the seventh volume of the first series of the Nouvelles Annales de Mathématiques, the study of quadric surfaces gave early rise to certain surfaces of the fourth order whose theory is closely related to that of quadrics, the normopolar surface and the first pedal of the ellipsoid (which is, in fact, none other than the surface of elasticity) being illustrations. Chief among such surfaces and the one most in- 
teresting, both to the mathematician and to the physicist, is the wave surface. Though one cannot say that this surface was distinctly mentioned by Fresnel prior to his memoir read before the French Academy in 1827, it is, nevertheless, true that it was hinted at in three previous papers of his, the first appearing in 1815 ; I have, therefore, considered myself justified in heading the list of references to articles upon the wave surface with references to these three papers. This surface has appealed distinctly to English investigators, fully one-half of the 84 articles upon the wave surface being from the pens of English mathematicians and physicists. This list includes Hamilton, Maccullagh, Lloyd, Lubbock, Sylvester, Archibald Smith, Moon, Cayley, Weddle, Walton, Prescott, W. Roberts, Tait, Turnbull, Niven, Townsend, Hicks, S. Roberts, Heavyside and Larmor. Among the long list of articles upon the wave surface, the length of which list may be considered remarkable when one considers that a single quartic surface is being treated, the fourteen papers of Mannheim, extending from 1867-1885 are to be especially noted. It would appear to be a most meritorious piece of work to compile the results embraced in this long list of papers, representing the investigations of three-quarters of a century, into a full and concise treatise upon the wave surface.

The recent appearance of the second edition of Darboux's memoir on cycliques and cyclides, a very able and interesting review of which, by Mr. Roberts, was published in the last May number of the Bulletin, shows the maintenance of deep interest in the anallagmatic surfaces of the fourth order. In the third volume of the Proceedings of the London Mathematical Society, page 189, the late Professor Cayley says that the Cyclide was first considered by Dupin in his "Applications de Géométrie, etc.," Paris, 1821, as the envelope of a sphere touching three given spheres; but as early as 1809, Dupin, in an article "Mémoire sur la sphère tangente à trois ou à quatre autres," published in the Correspondance sur l'École Polytechnique, hinted at this surface; and articles by Hachette, Binet, Gergonne and Poncelet, published between the years 1814 and 1820, upon the same subject, might be mentioned in the same connection. The chief honor, however, due for the present theory of anallagmatic surfaces of the fourth order, must be divided between Darboux and Casey, each of whom appears to have labored and achieved independently of the other.

In this bibliography, I have deemed it expedient, in order to avoid confusion, to separate the torus, which can be gen- 
erally defined as the surface generated by the revolution of a conic around a fixed axis anywhere situated, from the cyclide proper, and also to treat the remaining anallagmatic surfaces of the fourth order separately. Bordoni's article in the eighth volume of the Giornale di Fisica, Chimica $e$ Storia Naturale is the earliest paper that I find upon the torus proper.

Still another surface separates itself from the mass of quartic surfaces as possessing unusual interest, both intrinsically and historically, viz., Kummer's surface. This surface, being the 16-nodal quartic, gave immediate rise to the problem of the determination of the formula for the expression of the maximum number of nodes possessed by an $n$-thic surface, a problem that is, as far as I know, yet unsolved; the wave surface is, in fact, a particular case of Kummer's surface, in which but four of the nodes are real. Kummer's surface also bears an interesting relation to the theory of group transformations.

Steiner's surface, first determined by him as the Hessian of the general cubic surface, and the surface of elasticity, which I have already noted as being the first pedal of the ellipsoid, have each received enough attention to entitle them to be placed in a separate class.

Altogether, section 11 contains 390 references.

Section 12, treating of surfaces of the fifth and higher orders, need not be touched upon, except to mention that a nearly complete bibliography of quintic surfaces is given in a foot-note to the memoir, "On Quintic Surfaces," published in the first volume of The Mathematical Review and that the surfaces of this section, in so far as they have yet been studied, are mostly ruled surfaces, special developables, surfaces representable upon a plane and studied from that standpoint, and surfaces whose theory is inseparably connected with the theory of quadric surfaces. Among such last may be mentioned the parabolic ring of order six; the elliptic ring of order eight; the parallel surface of the paraboloid and the first central negative pedal of the ellipsoid of order ten; and the centro-surface of the ellipsoid and the parallel surface of the ellipsoid, of order twelve; all of which surfaces are remarkable in that their reciprocals are octadic 8-nodal quartic surfaces of the same species as the general torus.

The first reference to articles upon surfaces of revolution, other than quadrics, is that to an anonymous contribution to the seventh volume of the Annales de Mathématiques pures et appliquées, entitled "Caractères des surfaces de révolu- 
tion, ***," 1816. To this section there are sixty-seven references which, for the most part, treat of special surfaces. Articles by De la Gournerie, in the twentieth volume of the Journal de l'École Polytechnique and by Codazzi in the first volume of the Annali li Matematica are to be especially mentioned among the earlier contributions, while the papers of Pirondini (Nouvelles Annales de Mathématiques, 1888; Annali di Matematica, 1890 and 1894) may be noted from among those of the last few years. There are some seventy articles listed in this section.

Euler's article in the 7th volume of the Mémoires de l'Académie des Sciences de St. Pétersbourg, 1820, is the subject of the first reference in section 14 . Lamé's note and memoir in the Journal de Mathématiques pures et appliquées, 1838 and 1843 and Bertrand's paper in the Journal de l'École Polytechnique, 1843 follow. The subject of orthogonal surfaces is so closely connected with the consideration of isothermal surfaces that most articles treating of the one deal also with the other. The papers of Lamé and Bertrand, mentioned above, are, of the earlier papers, to be especially mentioned. There are 82 references to this section.

Of the 27 articles referred to in section 15 (envelopes), Livet's, in the first volume of the Correspondance sur l'École Polytechnique, 1803, followed closely by Monge's article in the fifth volume of the Journal de l'École Polytechnique, 1806, is the first to which reference is made. The envelopes treated are nearly all envelopes of planes or of spheres.

Of the 16 articles, references to which comprise the section upon reciprocal surfaces and which is introduced by reference to Salmon's paper in the $2 d$ volume of the Cambridge and Dublin Mathematical Journal, the several contributions of Salmon and Cayley, together with the notable paper of Zeuthen in the 10th volume of the Mathematische Annalen, are the worthiest.

References to articles upon the important subject of minimal surfaces (section 17), number 90. This section is introduced by reference to Gergonne's "Considerations preliminaires," published in the 7th volume of the Annales de Mathématiques pures et appliquées, 1816. Among the articles referred to, those by Schwarz (extending from 1865 to date), Lie and Enneper deserve a special mention. A minimal surface of peculiar interest is Enneper's celebrated surface of the ninth order (cf. Zeitschrift für Mathematik und Physik, volume 9). An interesting historical sketch and rapid review of minimal surfaces by Dr. Hancock is now running through The Mathematical Review. 
To articles upon ruled surfaces in general there are ninety reierences, the first being to Binet's paper in the second volume of the Correspondance sur l'École Polytechnique, 1809. The first strictly epoch-making paper, however, is that of Chasles, in the eleventh volume of the Correspondance mathématique et physique (Quetelet), 1839, pp. 49-113, entitled "Sur les surfaces engendrés par un ligne droite." There is, however, an important paper of date earlier than this, viz., 1826, that has been, as far as I know, overlooked. I refer to the paper of Karal Hube, "Dalcy ciag, etc." (On the formation of curved surfaces by a right line), published in the eleventh volume of the Rocznik Towarzystwa Nankowegoz Uniwersystetem Krakowskim Polaczonego.

Olivier's three articles in the Bulletin des Sciences de la Societé Philomathique de Paris, 1832-1833 and the Extraits des Procés-Verbaux des Séances de la Société Philomathique, 1838, are the first articles that I find treating specifically of scrolls. Of the fifty articles referred to in this division of section 18, the papers by Cayley, "On Skew Surfaces, otherwise Scrolls" (Cambridge and Dublin Mathematical Journal, volume 7 and Philosophical Transactions of the Royal Society of London, volumes 153, 154 and 159) are to be noted not only as being the first English contributions, but as epochmaking in themselves.

Of torses in general (not noting special developables), Poisson's articles, "Sur les surfaces developables," published in the Bulletin des Sciences de la Société Philomathique de Paris, 1825, and in the first volume of Baumgartner's Zeitschrift, 1826, are the earliest I find. Of the 60 articles listed in this division, many treat of special developables.

Jellett's paper, "Sur la surface dont la courbure moyenne est constante," in the Journal de Mathématiques pures et appliquées, 1853, is the first of the 30 papers referred to as treating specifically of surfaces of constant curvature. Lie's papers in the fourth and fifth volumes of the Tidsschrift for Mathematik are the ones to be especially noted.

Of the many special studies of geometry that have, from time to time, attracted the French school and to which it has attached itself, with its customary ardor, for a longer or shorter period of time, none has been more pronounced and noticeable than the study of surfaces defined by their lines of curvature. Attracted to the subject early in 1853, Bonnet had a 200-page memoir in Journal de l'Ecole Polytechnique and J. A. Serret a 50-page paper in the Journal de Mathématiques pures et appliquées before the year was over; and, to this day, the subject has been an engrossing one to 
the French school. Of English contributions, Cayley's paper in the eleventh volume of the American Journal of Mathematics might be mentioned.

Section 20, treating of special surfaces, contains references to all articles upon surfaces not included in previous sections. To this section, containing 340 references, space for but few remarks can be given. Applicable surfaces are introduced by Poisson's celebrated papers upon elastic surfaces, inserted in the third volume of the Correspondance sur l'École Polytechnique, 1812, the Mémoires de la classe des sciences mathématiques et physiques de l'Institut, 1812 and the Bulletin des Sciences de la Société Philomathique de Paris, 1814. The papers by Weingarten in the fifty-ninth volume of the Journal für die reine und angewandte Mathematik, the Nachrichten von der Gesellschaft der Wissenschaften zu Göttingen for 1887, and the one hundred and twelfth volume of the Comptes rendus hebdomadaires, as well as Codazzi's article in the Memoires preséntés par divers savants, etc., for 1883 should be also mentioned. 'The only English article I find is Cayley's review in the twenty-fourth volume of the Proceedings of the London Mathematical Society.

Of isothermal surfaces, Lamé's papers in the Annales de Chimie, 1833, the Journal de Mathématiques pures et appliquées. 1837, 1843, 1874, the Mémoires presentés par divers savants, etc., 1838 and Despeyron's memoir in the Journal für die reine und angewandte Mathematik, 1846 are to be especially remarked.

An article upon parallel surfaces dates back as far as 1813 , being Bordoni's "Sopra le $* * *$ superficie parallele" which appeared in the sixteenth volume of the Memorie dell' Accademia di scienze, lettere ed arti in Modena. S. Robert's articles in the fourth and fifth volumes of the Proceedings of the London Mathematical Society, 1873-1874, are the principal English contributions to the theory.

Normal surfaces are introduced by Brenner's paper in the thirteenth volume of the Archiv der Mathematik und Physik, and polar surfaces by Mainardi's article in the first volume of the Atti dell' Istituto Lombardo di scienze, lettere ed arti.

Of the rich literature upon pedal surfaces, there are to be especially mentioned the papers by W. Roberts in the tenth and twelfth volumes of the Journal de Mathématiques pures et appliquées, that of Tortolini in the thirty-first volume of the Journal für die reine und angewandte Mathematik and the articles by Hirst in the second volume of the Annali di Matematica, the third volume of The Quarterly Journal, the sixtysecond volume of the Journal für die reine und angewandte 
Mathematik and the twelfth volume of the Proceedings of the Royal Society of London. The origin of the theory goes back to Maclaurin, Philosophical Transactions of the Royal Society of London, 1718.

Concerning the remaining varieties of surfaces of this section space will permit no comment.

The general curve line, not lying in a single plane, was first studied by Pitot, 1728, who gave to such curves the name of curves of double curvature ; afterwards by Clairaut, 1731 ("Recherches sur les courbes à double courbure,") who gave expressions for the projections of these curves, for their tangents, normals, arc, etc.; by Monge, 1771 ("Mémoire sur les developées, etc.,") published 1785, in the tenth volume of the Mémoires présentées par divers savants, etc., as well as in his "Applications de l'analyse à la géométrie," who gave expressions for the normal plane, center and radius of curvature, evolutes, polar lines and polar developable, center of osculating sphere, for the criterion for points of simple inflexion (where four consecutive points are in one plane) and for points of double inflexion (where three consecutive points lie upon one straight line); by Tinseau, 1774("Solutions de quelques problèmes, etc. " published in the ninth volume of the Mémoires preséntées par divers savants, etc., 1780), who there considered the osculating plane* and the developable generated by the tangents; by Lacroix, in his Calcul Differentiel, 1790, who was the first to render the formulæ symmetrical by the introduction of the differentials of the three coördinates. The first reference of the century in this bibliography (section 21) is that to the memoir of Lancret (Mèmoire sur les courbes à double courbure") 1802, published in the first volume of the Mémoires $* * *$ savants étrangers de l'Institut, who calculated the angle of torsion and introduced the consideration of the rectifying lines and the rectifying surfaces. For further historical detail, the reader is referred to the able and admirable memoir of St. Venant (from which most of the above facts are taken), inserted in the eighteenth volume of the Journal de l'École Polytechnique, 1845. Of later papers, those of W. Schell, "Allgemeine Theorie der curven doppelter Krümmung in rein geometrischen Darstellung," Leipzig, 1859, and P. Serret, "Theorie nouvelle géométrique et mécanique des lignes à double courbure," Paris, 1860 are to be especially mentioned. Enne-

* The osculating plane, however, had been previously considered as far back as 1728, by John Bernouilli in his discussion of the problem : "In superficie quacunque curva ducere lineam inter duo puncta brevissimam," (of. his Opera omnia, volume 4, p. 113). 
per's paper in the Nachrichten von der Gesellschaft der Wissenschaften zu Göttingen, 1881, and Valentiner's in the second volume of the Acta Mathematica might be also remarked. This sections contains over 300 references.

Section 22 contains 40 references and section 23 contains 87 references.

Twisted cubics appear to have been first noted by Möbius in his classic Barycentric Calculus, 1827, and the first paper listed in this section is Poncelet's contribution to the seventh volume of the Correspondance mathématique et physique, 1832. Other papers to be noted are the thirty-third note of Chasles Aperçu historique, his article in the Journal de Mathématiques pures et appliquées, 1857, Schröter's contribution to the fifty-sixth volume of the Journal für die reine und angewandte Mathematik (as well as his work, published by Teubner in Leipzig, 1880), and the papers of Cremona in the first, second and fifth volumes of the Annali di Matimatica, and in the fifty-eighth, sixtieth and sixty-third volumes of the Journal für die reine und angewandte Mathematik; to these should be added the articles by Sturm in the seventy-ninth and eightieth volumes of the last mentioned Journal and the fortieth volume of the Zeitschrift für Mathematik und Physik, 1894, the papers by G. Loria (Rendiconti dell' Accademia della scienze, etc., Napoli, volume 29, 1885), by Reye (Mitteilung der Hamburger Mathematischen Gesellschaft, 1880), by Klein (Annali di Matematica, 1891) and by Valeri (Memorie dell' Accademia di scienze, lettere ed arti in Modena, $1892)$; this last paper, as well as the last mentioned paper by Sturm, deals with metric properties. The papers of Cayley in the ninth and tenth volumes of the Journal de Mathématiques pures et appliquées, 1844-1845, of Salmon in the forty-second volume of the Journal für die reine und angewandte Mathematik, 1851, of Hamilton in the eighth volume of the Proceedings of the Irish Academy, 1865, of Spottiswoode in the Proceedings and the Philosophical Transactions of the Royal Society of London, 1881, and of Dixon in the twentythird and twenty-fourth volumes of The Quarterly Journal, 1889, are the principal English contributions. There are 111 references to section 24 .

Twisted quartics of the first kind, being the intersection of two quadric surfaces, were naturally the first general species of twisted curves studied, and their history is identical with the history of quadric surfaces. The first article, reference to which is made in this section, is one by Hachette, inserted in the first volume of the Correspondance sur l'École Polytechnique, 1804 (Des courbes du quatrième de- 
gré, etc.). Among articles upon twisted quartics of the first kind that deserve especial mention, may be noted the papers of Chasles, found in the fifty-fourth and fifty-fifth volumes of the Comptes rendus hebdomadaires, 1862, of Tortolini, in the fifth volume of the Annali di Matematica, 1863, of Painvin, in the Nouvelles Annales de Mathématiques, volume 7, 1868, of Reye, Annali di Matematica, volume 2, 1876, of Weyr, Sitzungsberichte der math.-natur. Klasse der Akademie zu Wien, 1876, 1877 and 1878, of Schröter, Journal für die reine und angewandte Mathematik, 1882 (and in his "Grundlage einer reingeometrischen Theorie der Raumcurven vierter Ordnung erster Species," Leipzig, B. G. Teubner, 1890), of Lange, Zeitschrift für Mathematik und Physik, 1883 and of Milanowski, Journal für die reine und angewandte Mathematik, 1884.

Twisted quartics of the second kind were first shown to exist by Salmon, 1849, in his paper, "On the classification of curves of double curvature," inserted in the fifth volume of the Cambridge and Dublin Mathematical Journal, in which it was shown that every quartic of this kind always could be obtained as the residual intersection of a quadric surface and a cubic cone having one of the generators of the quadric for a double edge. Steiner independently arrived at this same fact in 1856 in his paper "Ueber die Flächen dritten Grades" published in the fifty-third volume of the Journal für die reine und angewandte Mathematik and in the Sitzungsberichte der Preussischen Akademie der Wissenschaften zu Berlin, 1856, Cremona's paper in the Rendiconti delle sessioni dell' Accademia delle scienze dell'Istituto di Bologna and the Annali di Matematica for 1861, Bertini's, in the Reale Istituto Lombardo di scienze $i$ lettere, Rendiconti, 1872, Eberhardt's, in the Zeitschrift für Mathematik und Physik 1877, Stahl's, in the Journal für die reine und angewandte Mathematik, volume 101 and Rohn's, in the forty-second and forty-third volumes of the Berichte über die Verhandlungen der Sächsischen Gesellschaft zu Leipzig are all papers worthy of particular mention. Altogether section 25 contains 106 references.

Of articles upon twisted quintics, those by Bertini ("In Memoriam Dominici Chelini, Collectanea Mathematica." Höpli, 1881), by Weyr (Sitzungsberichte der math.-natur. Klasse der Academie zu Wien, volumes 90, 92 and 97), by Montesano (Rendiconti dell' Accademia delle scienze, etc., Napoli, 1888) and by Berzolari (Memorie del l'Accademia dei Lincei, 1893), are the principal.

The papers of Weyr (Comptes rendus hebdomadaires, volume 76 and Sitzungsberichte der math.-natur. Klasse der Academie zu Wien, volumes 99 and 100) and the paper of London 
(Mathematische Annalen, volume 44), are the principal contributions to the theory of the twisted sextic; with the exception of the first, which deals with the classification of sextic curves in space, these all treat of the twisted sextic of deficiency one.

Of papers upon twisted curves of orders higher than six, Weyr's article in the sixty-ninth volume of the Sitzungsberichte der math. natur. Klasse der Academie zu Wien ("Ueber Raumcurven siebenter Ordnung"), is the only one in which a general treatment is found. Section 26 contains, altogether, twenty-two references.

Section 27 (special forms of twisted curves), containing 140 references, embraces the list of all articles, references to which could not find a fit place in the preceding sections.

To the observant student, such a bibliography as the one here briefly sketched, is interesting in many ways other than those at first apparent. It shows the trend of the different schools and their exoduses from one system or line of thought to another; the swarming of disciples after masters as the latter have changed the directions or methods or their researches ; the fluctuating enthusiasm in progressive fields of investigation; the numerous accidental duplications of discoveries and demonstrations, especially between different schools and languages; and, most important of all, it lays bare the "rag ends" that the masters, grown tired of one field of investigation, have left convenient for the disciple to lay hold of and unravel. Finally, it is believed, that to know well that which has already been done best fits one to do that which is, as yet, undone.

COLUMBIA UNIVERSITY.

\section{LINEAR DIFFERENTIAL EQUATIONS.}

Handbuch der Theorie der linearen Differentialgleichungen. Von Professor Dr. Ludwig Schlesinger, Privatdocenten an der Universität zu Berlin. In zwei Bänden. Erster Band. Leipzig, Teubner, 1895. 8vo, xx+486 pp.

The literature of the theory of linear differential equations has increased so rapidly during the last quarter of a century, and much of it has become of such importance that a detailed and methodical presentation of the subject such as is contained in the volume before us will be welcomed by large numbers of mathematicians and advanced students. While the author has attempted to make the 